\title{
New Exact Solutions of a Perturbed Nonlinear Schrodingers in Nonlinear Optics
}

\author{
M. A. Abdou ${ }^{1,2}$ - A. A. Soliman ${ }^{3,4}$
}

Published online: 11 May 2018

(C) The Author(s) 2018

\begin{abstract}
Here, new exact travelling wave solutions of a perturbed nonlinear Schrodingers equation arising in nonlinear optics are successfully obtained via the generalized Kudryashov method. The proposed method has been successfully implemented to seek exact solutions for the modeles coming for describing nonlinear optics. The results obtained by the generalized Kudryashov method is straightforward and concise mathematical tool to establish the exact analytical solutions of nonlinear equations. The solutions obtained here are new and have not been reported in former literature.
\end{abstract}

Keywords Perturbed nonlinear Schrodingers equation (NLSE) · The generalized Kudryashov method $\cdot$ New exact solutions

\section{Introduction}

The investigation of the travelling wave solutions for nonlinear evolution equations arising in mathematical physics plays an important role in the study of nonlinear physical phenomena. The nonlinear evolution equations are major subjects in physical science,appears in various scientific and engineering fields, such as fluid mechanics, plasma physics, optical fibers, biology, solid state physics, chemical kinematics, chemical physics and ochemistry. Nonlinear wave phenomena of dissipation, diffusion, reaction and convection are very important in

$凶 \quad$ M. A. Abdou

m_abdou_eg@yahoo.com

1 Physics Department, College of Science, University of Bisha, PO Box 344, Bisha 61922, Kingdom of Saudi Arabia

2 Theoretical Research Group, Physics Department, Faculty of Science, Mansoura University, Mansoura 35516, Egypt

3 Department of Mathematics, College of Science, University of Bisha, PO Box 344, Bisha 61922, Kingdom of Saudi Arabia

4 Department of Mathematics, Faculty of Science, AL-Arish University, AL-Arish 45111, Egypt 
nonlinear wave equations. In the past several decades,new exact solutions may help to find new phenomena. A variety of powerful methods for obtaining the exact solutions of nonlinear evolution equations have been presented [1-23].

The nonlinear Schrodinger equation has a central importance in many natural sciences as well as engineering with numerous interpretations and applications concerning e.g. nonlinear optics, protein chemistry, plasma physics and fluid dynamics. This paper will consider the perturbed NLSE which governs the dynamics of solitons in negativeindex material with nonKerr nonlinearity and third-order dispersion, and the dimensionless form of the equation is given by [24-29]

$$
\begin{aligned}
& i u_{t}+a u_{x x}+b u_{x t}+c F\left(|u|^{2}\right) u=-i d u_{x}-i s\left(|u|^{2} u\right)_{x}-i \mu\left(|u|^{2}\right)_{x} u-i \theta|u|^{2} u_{x} \\
& \quad-i \gamma u_{x x x}-\theta_{1}\left(|u|^{2} u\right)_{x x}-\theta_{2}|u|^{2} u_{x x}-\theta_{3} u^{2} u_{x x}^{*}
\end{aligned}
$$

where $u(x, t)$ is the complex field amplitude. $a, b$, and $c$ are the coefficients of group velocity dispersion, spatial temporal dispersion and non-Kerr nonlinearity, and $d, s, \mu, \theta$ and $\gamma$ account for the inter-modal dispersion, selfsteepening, Raman effect, nonlinear dispersion and third order dispersion, respectively. The last three terms appear in the context of negative-index material.

The main aim of this study is to extract exact solitons to Eq. (1) using the proposed method. Four different kinds of nonlinearity are considered for Eq. (1). They are Kerr law, power law, parabolic law and dual-power law.

\section{Analysis of the Method}

In what follows the properties of the the generalized Kudryashov method [30,31] as:

For a given the general nonlinear partial differential equation of the type

$$
\psi\left(u, u_{t}, u_{x}, u_{x x}, u_{t t}, \ldots\right)=0, t>0
$$

where $u(x, t)$ is an unknown function, $x$ is the spatial variable and $t$ is the time variable, $\psi$ is a polynomial in $u$ and its derivatives, in which the highest order derivatives and nonlinear terms are involved.

Step 1 The travelling wave variable $\xi=x-v t$ transform Eq. (2) into ODE as

$$
\phi\left(u, u^{\prime}, u^{\prime}, u^{\prime \prime}, \ldots\right)=0
$$

where the prime denotes to the differenation with respect to $\xi$.

Step 2 Considering trial equation of solution in Eq. (3), it can be written as

$$
U(\xi)=\frac{\sum_{i=0}^{n} a_{i} Q^{i}(\xi)}{\sum_{j=0}^{m} b_{j} Q^{j}(\xi)}=\frac{A[Q(\xi)]}{B[Q(\xi)]}
$$

where $A$ and $B$ are polynomial of $Q(\xi)$.

Therefore, we can find the value of $N$ and $M$, where $Q=Q(\xi)$ satisfies the following ODE:

$$
Q^{\prime}(\xi)=Q^{2}(\xi)-Q(\xi)
$$


The Riccati Eq. (5) admits the following exact solution as follows:

$$
\begin{array}{ll}
Q_{1}(\xi)=\frac{1}{2}\left[1-\tanh \left[\frac{\xi}{2}-\frac{\epsilon \ln \xi_{0}}{2}\right]\right], & \xi_{0}>0 \\
Q_{2}(\xi)=\frac{1}{2}\left[1-\operatorname{coth}\left[\frac{\xi}{2}-\frac{\epsilon \ln \xi_{0}}{2}\right]\right], & \xi_{0}<0
\end{array}
$$

where $\epsilon$ is an constant.

Step 3 The positive integer $N$ and $M$ appearing in Eq. (4) can be determined by considering the balancing between the highest order derivative and the nonlinear term comes from Eq. (2) via the relations

$$
\begin{aligned}
D\left[\frac{\partial^{q} u}{\partial \xi^{q}}\right) & =N-M+q, \\
D\left[u^{p}\left(\frac{\partial^{q} u}{\left.\partial \xi^{q}\right)^{s}}\right)\right) & =(N-M) p+s(N-M+q),
\end{aligned}
$$

where $p, q, s$ are integer numbers. Therefore, we can find the value of $N$ and $M$ in Eq. (4).

Step 4 Inserting Eq. (4) along Eq. (5) into Eq. (3) and collecting all the terms of the same power $Q^{i}(\xi),(i=0,1,2, \ldots)$ and equating them to zero, we obtain a system of algebraic equations, which can be solved it for obtaning the values $A_{i}, B_{j}, c, \delta$. Substituting the values in Eq. (4) along with general solutions of Eq. (5) one can directly evaluated the exact solutions of Eq. (9).

\section{The New Exact Solutions of Eq. (1)}

To solve Eq. (1), we use the wave transformations as

$$
u(x, t)=P(\xi) e^{i \phi(x, t)},
$$

where $P(\xi)$ represents the shape of the pulse and

$$
\xi=x-v t, \phi(x, t)=-k x+w t+\xi_{0}
$$

In Eq. (9), $\phi(x, t)$ gives the phase component of the soliton. Then, in Eq. (10), $k, w$ and $\xi_{0}$ respectively represent the frequency, wave number and phase constant and in Eq. (10), $v$ shows the velocity of the soliton.

By using Eq. (9) into Eq. (1) and then decomposing into real and imaginary parts yield a pair of relations. Real part gives

$$
\begin{aligned}
& (a-b v+3 k \gamma) P^{\prime \prime}-\left((1-b k) w+a k^{2}-d k+\gamma k^{3}\right) P \\
& \quad+c F\left(P^{2}\right) P+\left(s k+\theta k-\theta_{1} k^{2}-\theta_{2} k^{2}-\theta_{3} k^{2}\right) P^{3} \\
& \quad+6 \theta_{1} P\left(P^{\prime 2}\right)+\left(3 \theta_{1}+\theta_{2}+\theta_{3}\right) P^{2} P^{\prime \prime}=0,
\end{aligned}
$$

and the maginary part reads

$$
\left(-v-2 a k+b w+b k v+d-3 \gamma k^{2}\right) P^{\prime}+\left(3 s+2 \mu+\theta-2 k\left(3 \theta_{1}+\theta_{2}-\theta_{3}\right) P^{2} P^{\prime}+\gamma P^{\prime \prime \prime}=0\right.
$$

From the maginary part, we have

$$
\gamma=0, v=-\frac{2 a k-b w-d}{1-b k}, \quad 3 s+2 \mu+\theta-2 k\left(3 \theta_{1}+\theta_{2}-\theta_{3}\right)=0
$$


With aid of Eq. (13), with $\theta_{1}=0, \theta_{2}=-\theta_{3}$ and $s=-\theta$, Eq. (12) yields

$$
(a-b v) P^{\prime \prime}-\left((1-b k) w+a k^{2}-d k\right) P+c F\left(P^{2}\right) P=0,
$$

where $\theta-\mu-2 \theta_{3} k=0$

\section{For Kerr Law}

For Kerr law nonlinearity as $F(q)=q$, then Eq. (14) becomes

$$
(a-b v) P^{\prime \prime}-\left((1-b k) w+a k^{2}-d k\right) P+c P^{3}=0
$$

In this section the propsed methed will be used for construction the new exact solution of Eq. (15). Now balancing the highest order derivative $P^{\prime \prime}$ and nonlinear term $P^{3}$, we get $3 N-3 M=N-M+2$ or equivalent $N=M+1$. Setting $M=1$, we obtain $N=2$. Then Eq. (14) reads

$$
P(\xi)=\frac{a_{0}+a_{1} Q(\xi)+a_{2} Q^{2}(\xi)}{b_{0}+b_{1} Q(\xi)}
$$

Making use of Eq. (16) into (15) with Eq. (5), and collecting all power of $Q(\xi)$, we get a system of algebraic equations. By solving this algebraic system of equations, we have

case (1)

$$
\begin{gathered}
k=k, \quad v=\frac{\left(a b_{1}^{2}+2 c a_{1}^{2}\right)}{b b_{1}^{2}}, \quad w=\frac{\left(-d k b_{1}^{2}+a k^{2} b_{1}^{2}-c a_{1}^{2}\right)}{b_{1}^{2}(-1+b k)}, \\
a_{0}=0, \quad a_{1}=a_{1}, \quad a_{2}=-2 a_{1}, \quad b_{0}=0, \quad b_{1}=b_{1}
\end{gathered}
$$

case (2)

$$
\begin{gathered}
k=k, \quad v=\frac{\left(2 c a_{0}^{2}+a b_{0}^{2}\right)}{b b_{0}^{2}}, \quad w=\frac{\left(a k^{2} b_{0}^{2}-c a_{0}^{2}-d k b_{0}^{2}\right)}{b_{0}^{2}(-1+b k)}, \\
a_{0}=a_{0}, \quad a_{1}=-\frac{\left(2 b_{0}+b_{1} a_{0}\right.}{b_{0}}, \quad a_{2}=0, \quad b_{0}=b_{0}, \quad b_{1}=b_{1}
\end{gathered}
$$

In view of Eq. (17), inserting Eq. (17) into (16), admits to the new exact solution of Eq. (15) as

$$
\begin{aligned}
P(\xi) & =\frac{a_{1} Q(\xi)-2 a_{1} Q^{2}(\xi)}{b_{1} Q(\xi)}, \\
u(x, t) & =P(\xi) e^{i \phi(x, t)} \\
\xi & =x-v t, \phi(x, t)=-k x+w t+\xi_{0}
\end{aligned}
$$

where $w, v$ and $k$ are given in Eq. (17),

$$
\begin{array}{ll}
Q_{1}(\xi)=\frac{1}{2}\left[1-\tanh \left[\frac{\xi}{2}-\frac{\epsilon \ln \xi_{0}}{2}\right]\right], & \xi_{0}>0 \\
Q_{2}(\xi)=\frac{1}{2}\left[1-\operatorname{coth}\left[\frac{\xi}{2}-\frac{\epsilon \ln \xi_{0}}{2}\right]\right], & \xi_{0}<0
\end{array}
$$

Remark 1 it is worth noting the new exact solutions obtained here are new and have not been reported in former literature. For simplicity case (2) should be omitted here. 


\section{For Power Law}

In this case, we assume as $F(q)=q^{n}$, then Eq. (14) becomes

$$
(a-b v) P^{\prime \prime}-\left((1-b k) w+a k^{2}-d k\right) P+C P^{2 n+1}=0
$$

In the same manner, to solve for obtaining the exact solution of Eq. (24), we use

$$
P=u^{\frac{1}{2 n}}
$$

Then Eq. (24) reads

$$
(a-b v)\left((1-2 n) U^{\prime 2}+2 n U U^{\prime \prime}\right)-4 n^{2}\left((1-b k) w+a k^{2}-d k\right) U^{2}+4 c n^{2} U^{3}=0
$$

Balancing the highest order derivative $U U^{\prime \prime}$ and nonlinear term $U^{3}$, we get $3 N-3 M=$ $N-M+2$ or equivalent $N=M+2$. For $M=1$, we obtain $N=3$. Then Eq. (26) reads

$$
U(\xi)=\frac{a_{0}+a_{1} Q(\xi)+a_{2} Q^{2}(\xi)+a_{3} Q^{3}(\xi)}{b_{0}+b_{1} Q(\xi)}
$$

Inserting Eq. (27) into (26) with Eq. (5), and collecting all power of $Q(\xi)$, we get a system of algebraic equations. By solving this algebraic system of equations, we have

case (1)

$$
\begin{aligned}
k & =k, \quad v=v, \quad w=w, \quad a_{0}=\frac{b_{0}\left(w-w b k+a k^{2}-d k\right)}{c}, \\
a_{1} & =\frac{b_{1}\left(w-w b k+a k^{2}-d k\right)}{c}, \quad a_{0}=0, \quad a_{3}=0, \\
b_{0} & =b_{0}, \quad b_{1}=b_{1}
\end{aligned}
$$

case (2)

$$
\begin{aligned}
& v=-\frac{\left(-a+4 n^{2} w-4 n^{2} w b k+4 n^{2} a k^{2}-4 n^{2} d * k\right)}{b}, \\
& a_{1}=\frac{4 b_{0}\left(-n w b k+n a k^{2}-n d k+n w+w-w b k+a k^{2}-d k\right)}{c}, \\
& a_{2}=\frac{-4\left(-n d k b_{0}-n b_{1} w-n b_{1} a k^{2}+n b_{1} w b k+n w b_{0}+n b_{1} d k-n w b k b_{0}+n a k^{2} b_{0}-d k b_{0}-w b_{1}-a k^{2} b_{1}+w b k b_{1}+w b_{0}+d k b_{1} w b k b_{0}+a k^{2} b_{0}\right.}{c}, \\
& k=k, \quad w=w, \quad a_{0}=0, \quad b_{0}=b_{0}, \quad b_{1}=b_{1}
\end{aligned}
$$

Using Eq. (28) into (27), one can directly obtined the new exact traveling wave solution as

$$
\begin{aligned}
U(\xi) & =\frac{\frac{b_{0}\left(w-w b k+a k^{2}-d k\right)}{c}+a_{1} Q(\xi)+\left[\frac{b_{1}\left(w-w b k+a k^{2}-d k\right)}{c}\right] Q^{2}(\xi)}{b_{0}+b_{1} Q(\xi)}, \\
P & =U^{\frac{1}{2 n}} \\
u(x, t) & =P(\xi) e^{i \phi(x, t)}, \\
\xi & =x-v t, \phi(x, t)=-k x+w t+\xi_{0}
\end{aligned}
$$

where $w, v$ and $k$ are given in Eq. (28),

$$
\begin{aligned}
& Q_{1}(\xi)=\frac{1}{2}\left[1-\tanh \left[\frac{\xi}{2}-\frac{\epsilon \ln \xi_{0}}{2}\right]\right], \xi_{0}>0 \\
& Q_{2}(\xi)=\frac{1}{2}\left[1-\operatorname{coth}\left[\frac{\xi}{2}-\frac{\epsilon \ln \xi_{0}}{2}\right]\right], \xi_{0}<0
\end{aligned}
$$


Remark 2 The new exact traveling wave solution obtained here via the proposed method are new and have not been reported in former literature. For simplicity case (2) should be omitted here.

\section{For Parabolic Law}

Here, we set $F(q)=q+\eta q^{2}$, then Eq. (14) becomes

$$
(a-b v) P^{\prime \prime}-\left((1-b k) w+a k^{2}-d k\right) P+c P^{3}+c \eta P^{5}=0
$$

To solve the reduced Eq. (36) for constructing the new exact solutions of Eq. (36), we use

$$
P=U^{\frac{1}{2}}
$$

Then Eq. (36) can be rewritten as

$$
(a-b v)\left(2 U U^{\prime \prime}-U^{\prime 2}\right)-4\left((1-b k) w+a k^{2}-d k\right) U^{2}+4 c U^{3}+4 c \eta U^{4}=0
$$

Balancing the highest order derivative $U U^{\prime \prime}$ and nonlinear term $U^{4}$, we get $N=M+1$ and

$$
U(\xi)=\frac{a_{0}+a_{1} Q(\xi)+a_{2} Q^{2}(\xi)}{b_{0}+b_{1} Q(\xi)}
$$

Inserting Eq. (39) into (38) with Eq. (5), and collecting all power of $Q(\xi)$, we get a system of algebraic equations. By solving this algebraic system of equations, we have

$$
\begin{aligned}
c & =\frac{-3 b_{1}^{2}\left(w-w b k+a k^{2}-d k\right)}{a_{2} \eta}, \quad k=k, \\
v & =-\frac{\left(-4 d k-a-4 w b k+4 a k^{2}+4 w\right)}{b}, \quad w=w, \quad a_{0}=0, \\
a_{1} & =-a_{2}, \quad a_{2}=a_{2}, \quad b_{0}=\frac{-1}{8} \frac{b_{1}\left(4 a_{2} \eta-3 b_{1}\right)}{a_{2} \eta}, \quad b_{1}=b_{1}
\end{aligned}
$$

Making use Eq. (40) into (39), admits to

$$
\begin{aligned}
U(\xi) & =\frac{-a_{2} Q(\xi)+a_{2} Q^{2}(\xi)}{\frac{-1}{8} \frac{b_{1}\left(4 a_{2} \eta-3 b_{1}\right.}{a_{2} \eta}+b_{1} Q(\xi)}, \\
P & =U^{\frac{1}{2}}, \\
u(x, t) & =P(\xi) e^{i \phi(x, t)} \\
\xi & =x-v t, \phi(x, t)=-k x+w t+\xi_{0}
\end{aligned}
$$

where $w, v$ and $k$ are given in Eq. (40),

$$
\begin{array}{ll}
Q_{1}(\xi)=\frac{1}{2}\left[1-\tanh \left[\frac{\xi}{2}-\frac{\epsilon \ln \xi_{0}}{2}\right]\right], & \xi_{0}>0 \\
Q_{2}(\xi)=\frac{1}{2}\left[1-\operatorname{coth}\left[\frac{\xi}{2}-\frac{\epsilon \ln \xi_{0}}{2}\right]\right], & \xi_{0}<0
\end{array}
$$

Remark 3 The new exact solutions Eq. (41) obtained here are new and have not been reported in former literature. 


\section{Conclusion and Summary}

In this work, we investigated analytically solutions the nonlinear physical model Eq. (1) arising in nonlinear optics vis the generalized Kudryashov method. Different kinds of nonlinearities including Kerr law, power law, parabolic law and dual-power law are taken into accountd.

The generalized Kudryashov method has been successfully implemented to seek exact solutions for nonlinear differential equations coming for describing nonlinear optics. An extended methods will be used such as [32-41] for constructing the travelling wave solutions of a fractional order space-time nonlinear evolution equations arising in nonlinear optics. This our task in future.

We conclude that the studied method can be more effectively applied to investigate other nonlinear partial differential equations which frequently arise in mathematical physics and other scientific application fields.

To our knowledge, these new solutions have not been reported in former literature, they may be of significant importance for the explanation of some special physical phenomena.

Acknowledgements This paper have been supported by research support program,University of Bisha,Kingdom of Saudi Arabia, Grant Number (UB-14-1438) and (UB-09-1438). The authors would like to express their great thankful to the referees for their useful comments.

Open Access This article is distributed under the terms of the Creative Commons Attribution 4.0 International License (http://creativecommons.org/licenses/by/4.0/), which permits unrestricted use, distribution, and reproduction in any medium, provided you give appropriate credit to the original author(s) and the source, provide a link to the Creative Commons license, and indicate if changes were made.

\section{References}

1. Ablowitz, M., Clarkson, P.A.: Soliton, Nonlinear Evolution Equations and Inverse Scattering. Cambridge University Press, New York (1991)

2. El-Wakil, S.A., Abdou, M.A.: Modified extended tanh function method for solving nonlinear partial differential equations. Chaos Solitons Fractals 31, 1256-1264 (2007)

3. El-Wakil, S.A., Abdou, M.A.: New exact travelling wave solutions of two nonlinear physical models. Nonlinear Anal. 68, 235-245 (2008)

4. Ping, L.C.: $\left(\frac{G^{\prime}}{G}\right)$-expansion method equivalent to extended tanh function method. Commun. Theor. Phys. 51, 985-988 (2009)

5. Peng, Y.: Traveling wave-like solutions of the Zakharov-Kuznetsov equation with variable coefficients. J. Phys. 71, 49-55 (2008)

6. Wazwaz, A.M.: New solitons and kinks solutions to the Sharma-Tasso-Olver equation. Appl. Math. Comput. 188, 1205-1213 (2007)

7. Zayed, E.M.E., Gepreel, K.A.: Some applications of the $\left[\frac{G^{\prime}}{G}\right]$-expansion method to non-linear partial differential equations. Appl. Math. Comput. 212, 1-13 (2009)

8. Zhang, H.: New application of the $\left[\frac{G^{\prime}}{G}\right]$-expansion method. Commun. Nonlinear Sci. Numer. Simul. 14, 220 (2009)

9. Wang, M., Li, X., Zhang, J.: The $\left[\frac{G^{\prime}}{G}\right]$-expansion method and travelling wave solutions of nonlinear evolution equations. Math. Phys. Phys. Lett. A 372, 417-423 (2008)

10. Ugurlu, Y.: Analytic method for solitary solutions of some partial differential equations. Phys. Lett. A 370(3-4), 251-259 (2007)

11. Abulwafa, E.M., Abdou, M.A., Mahmoud, A.A.: The solution of nonlinear coagulation problem with mass loss. Chaos Solitons Fractals 29, 313-330 (2006)

12. He, J.H., Wu, X.-H.: Exp-function method for nonlinear wave equations. Chaos Solitons Fractals 30, 700-708 (2006) 
13. Abdou, M.A.: Further improved F-expansion and new exact solutions for nonlinear evolution equations. J. Nonlinear Dyn. 52(3), 277-288 (2007)

14. Abdou, M.A.: Exact periodic wave solutions for some nonlinear evolution equations. Int. J. Nonlinear Sci. 5, 1-9 (2008)

15. Abdou, M.A., Zhang, S.: New periodic wave solutions via extended mapping method. Commun. Nonlinear Sci. Numer. Simul. 14, 2-11 (2009)

16. El-Wakil, S.A., Madkour, M.A., Abdou, M.A.: Application of Exp-function method for nonlinear evolution equations with variable coefficients. Phys. Lett. A 369, 62-69 (2007)

17. Zhou, Y., Wang, M., Wang, Y.: Periodic wave solutions to a coupled KdV equations with varable coeffients. Phys. Lett. A 308, 31-36 (2003)

18. Abdou, M.A.: On the variational iteration method. Phys. Lett. A. 366, 61-68 (2007)

19. El-Wakil, S.A., Abdou, M.A., Hendi, A.: New periodic wave solutions via Exp-function method. Phys. Lett. A 372, 830-840 (2008)

20. Abdou, M.A.: Generalized solitary and periodic solutions for nonlinear partial differential equations by the Exp-function method. J. Nonlinear Dyn. 52(1-2), 1-9 (2008)

21. El-Wakil, S.A., Abdou, M.A.: The extended mapping method and its applications for nonlinear evolutions equations. Phys. Lett. A 358, 275-282 (2006)

22. Li, B., Ma, Y., Sun, J.: The interaction processes of the N-soliton solutions for a extended generalization of Vakhnenko equation. App. Math. Comput. 216(12), 3522-3535 (2010)

23. Dai, Z., Li, Z., Liu, Z., Li, D.: Exact cross kink-wave solutions and resonance for the Jimbo-Miwa equation. Physica A 384, 285 (2007)

24. Zhou, Q., Liu, L., Liu, Y., Yu, H., Yao, P., Wei, C., Zhang, H.: Exact optical solitons in metamaterials with cubic-quintic nonlinearity and third-order dispersion. Nonlinear Dyn. 80(3), 1365-1371 (2015)

25. Zhou, Q., Mirzazadeh, M., Ekici, M., Sonmezoglu, A.: Analytical study of solitons in non-Kerr nonlinear negative-index materials. Nonlinear Dyn. 86, 623-638 (2016)

26. Biswas, A., Khan, K.R., Mahmood, M.F.: Bright and dark solitons in optical metamaterials. Optik 125(3), 3299-3302 (2014)

27. Xu, Y., Savescu, M., Khan, K.R., Mahmood, M., Biswas, A., Belic, M.: Soliton propagation through nanoscale waveguides in optical metamaterials. Opt. Laser Technol. 77, 177-186 (2016)

28. Saha, M., Sarma, A.K.: Modulation instability in nonlinear metamaterials induced by cubic-quintic nonlinearities and higher order dispersive effects. Optics Commun. 291, 321-325 (2013)

29. Yang, R., Zhang, Y.: Exact combined solitary wave solutions in nonlinear metamaterials. J. Opt. Soc. Am. B 28(1), 123-127 (2011)

30. Khan, K., Abaker M. A., Arnous, A.: Springer Plus 5, 663 (2016)

31. Kudryashov, N.A.: One method for finding exact solutions of nonlinear differential equations. Commun. Nonlinear Sci. Numer. Simul. 17(6), 2248-2253 (2012)

32. Abdou, M.A., Elgarayhi, A., Attia, A.T.: New exact solutions for space-time fractal order on the ion acoustic waves in electron-positron-ion plasma. Nonlinear Sci. Lett. A 5, 35-44 (2014)

33. Abdou, M.A., Elgarayhi, A., El-Shewy, E.: Fractional complex transform for space-time fractional nonlinear differential equations arising in plasma physics. Nonlinear Sci. Lett. A. 5, 31-34 (2014)

34. Abdou, M.A., Elhanbaly, A.: New application of the fractional sub-equation method. Nonlinear Sci. Lett. A 6(1), 10-18 (2015)

35. Abdou, M.A.: On the fractional order space-time nonlinear equations arising in plasma physics. Indian J. Phys. (2018) (in press)

36. Abdou, M.A., Yildirim, A.: Approximate analytical solution to time fractional nonlinear evolution equations. Int. J. Numer. Methods Heat Fluid Flow 22, 829-838 (2015)

37. Abdou, M.A.: An analytical method for space-time fractional nonlinear differentialequations arising in plasma physics. J. Ocean Eng. Sci. 2, 1-5 (2017)

38. Abdou, M.A., Soliman, A.A.: New exact travelling wave solutions for space-time fractional nonlinear equations describing nonlinear transmission lines. Results Phys. (2018) (in press)

39. Abdou, M.A., Soliman, A.A.: On the space-time fractal order on ion acoustic waves in electron-positonion plasma. Int. J. Appl. Math. Comput. Math. (2018) (in press)

40. Bin, L.: Backlund transformation of frcational Riccati equation and its applications to nolinear fractional partial differential equations. Phys. Lett. A 376, 2045-2048 (2012)

41. Feng, Q.: Exact solutions for fractional partial differential equations by a new generalized fractional subequation method. WSEAS Trans. Math. 15, 44 (2016) 